\title{
Cannabis und Schadensminderung in Deutschland
}

\section{Cannabis and Harm Reduction in Germany}

(๑)(1) $\odot$

\author{
Autoren \\ Jakob Manthey' ${ }^{1}$, Heino Stöver ${ }^{2}$, Hans-Günter Meyer-Thompson ${ }^{3}$
}

Institute

1 Institut für Klinische Psychologie und Psychotherapie, Technische Universität Dresden, Dresden

2 Institut für Suchtforschung (ISFF), Frankfurt University of Applied Sciences, Frankfurt

3 Ambulanz Altona, Klinik für Abhängigkeitserkrankungen, ASKLEPIOS Hamburg Nord Ochsenzoll, Hamburg

\author{
Schlüsselwörter \\ Cannabis, Schadensminderung, Drogenpolitik Drogen- \\ aufklärung, Deutschland
}

Key words

cannabis, harm reduction, drug policy, drug education, Germany

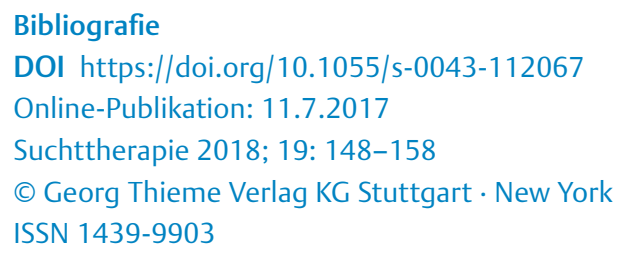

Korrespondenzadresse

Dipl.-Psych. Jakob Manthey

Institut für Klinische Psychologie und

Psychotherapie TU Dresden

Chemnitzer Straße 46

01187 Dresden

jakob.manthey@tu-dresden.de

\section{ZUSAMMENFASSUNG}

Aktuelle drogenpolitische Diskussionen über Cannabis drehen sich v. a. darum, wie diese Substanz in Zukunft rechtlich reguliert werden soll. Angesichts des anhaltend weit verbreiteten Konsums und der damit verbundenen Risiken, sollten jedoch mögliche Strategien zur Reduzierung der potenziellen Schäden für Konsumierende und die Gesellschaft bei der Gestaltung einer adäquaten Cannabispolitik berücksichtigt werden. Dieser Beitrag gibt einen Überblick über verschiedene Möglichkeiten zur Reduzierung der Konsumrisiken und illustriert, inwieweit diese bereits in Deutschland implementiert sind.

Es lassen sich sowohl individuelle als auch strukturelle Ansätze zur Reduzierung cannabisbezogener Risiken identifizieren. Individuelle Ansätze zielen durch die Vermittlung von Informationen darauf ab, dass Individuen durch eine risikoarme Konsumpraxis selbstständig die Risiken reduzieren und somit potenziell Schäden vermeiden. Strukturelle Ansätze dagegen sollen das Konsumumfeld so gestalten, dass der Konsum mit möglichst geringen negativen Folgen einhergeht. In Deutschland sind sowohl individuelle als auch strukturelle Ansätze zur Schadensminderung von Cannabiskonsum nicht in ausreichendem Maße umgesetzt. Mögliche Gründe für eine mangelhafte Implementierung werden diskutiert und Potenzial für die Anwendung von schadensmindernden Strategien für Cannabis identifiziert.

\section{ABSTRACT}

Current discussions on drug policy commonly focus on the future of the legal regulation of cannabis. Given the ongoing and prevalent use of cannabis and use-related risks, an adequate cannabis policy should consider available strategies to avoid possible harms for users and the society. This work gives an overview on various approaches to reduce use-related risks and describes the extent to which they are already implemented in Germany.

There are individual and structural approaches to reduce cannabis-related risks. Individual approaches promote information on safer use behaviors. Users following these advices can reduce risks and avoid potential harm. Structural approaches aim to alter the users' environment to minimize adverse consequences of cannabis use. In Germany, the implementation of both individual and structural approaches is very limited. Possible reasons for insufficient implementation and the potential to apply harm reduction strategies are discussed.

\section{Einleitung}

Cannabis ist die mit Abstand am weitesten verbreitete illegale Droge in Deutschland, wobei ca. 4.5 \% der erwachsenen Bevölke- rung angeben, im letzten Jahr Cannabis konsumiert zu haben [1-3]. Der Cannabiskonsum ist in der Altersgruppe der 12-17 (7,3\%) und 18-25 (16,3\%) Jährigen am verbreitetsten. In diesen 
Altersgruppen befindet sich zudem ein bedeutender Anteil regelmäßig Konsumierender (mind. 10-mal im letzten Jahr: 2,6\%). Über die letzten 15 Jahre unterlagen sowohl die 12-Monats-Prävalenzraten als auch der Anteil regelmäßig Konsumierender unter Jugendlichen und jungen Erwachsenen ähnlichen Schwankungen [3]. Der Konsum von Cannabis ist sowohl mit vielfältigen Genussaspekten, als auch mit vielen negativen gesundheitlichen Auswirkungen assoziiert [4-6]. Die negativen gesellschaftlichen Folgen des Cannabiskonsums spiegeln sich u. a. in einer erhöhten Belastung für das Gesundheitssystem und für Angehörige wider [7] und resultieren in einer nicht zu vernachlässigenden gesamtgesellschaftlichen Belastung (Ergebnisse für Kanada: [8]).

Vor diesem Hintergrund ist zu begrüßen, dass der Umgang mit Cannabis auch in Deutschland verstärkte Beachtung in der politischen und medialen Öffentlichkeit, aber auch in der fachlichen Diskussion findet (siehe z. B. Suchttherapie: Jahrgang 17, Ausgabe 2; SUCHT: Jahrgang 62, Ausgabe 1). Die Diskussion um die bestmögliche rechtliche Regulierung erfährt derzeit vermutlich die meiste Aufmerksamkeit (z. B. [9-11]), wobei positive Entwicklungen in anderen Feldern (z. B. in der Suchthilfe [12]) in dieser Diskussion oftmals untergehen. Im Rahmen der öffentlichen Diskussion wird sich wiederholt auf die Konsumprävalenz bezogen, welche neben üblicher Schwankungen $[1,13]$ auch durch den rechtlichen Status der Substanz beeinflusst werden kann [14-16]. Während die Unterbindung des Cannabiskonsums traditionell als Maßstab effektiver Drogenpolitik und Ziel jeglicher Präventionsmaßnahmen ausgegeben wurde, kann nach derzeitigen Erkenntnissen davon ausgegangen werden, dass die vollständige Eliminierung des Konsums kein realistisches Ziel darstellt. Diese Perspektive impliziert aber auch, dass die Risiken und negativen Folgen von Cannabiskonsum für Individuen und die Gesellschaft bestehen bleiben und dementsprechend bedacht werden müssen.

Die Ausrichtung drogenpolitischer Strategien auf die Reduzierung der individuellen und gesellschaftlichen Belastung durch Cannabiskonsum wäre im Sinne eines Public Health Ansatzes [17]. Hierbei werden Strategien zur Verhinderung und Reduzierung des Konsums durch Prävention und Repression auch künftig eine zentrale Rolle spielen. Diese sind jedoch kaum geeignet, um spezifische Risiken, denen sich Konsumierende aussetzen, zu reduzieren. Für dieses Ziel bedarf es schadensmindernder oder Harm Reduction (nachfolgend: HR) Strategien [18], welche bereits eine der 4 Ebenen deutscher Drogenpolitik repräsentiert [1]. HR Strategien werden in Deutschland bereits für verschiedene andere illegale Substanzen erfolgreich angewendet [19], jedoch fehlt bislang ein expliziter Bezug auf Cannabiskonsum [20] über präventive Ansätze hinaus.

Die vorliegende Arbeit soll daher mittels narrativem Review (1) den gegenwärtigen Forschungsstand zu Cannabis und HR beschreiben und (2) aufzeigen, in welchem Maß HR Prinzipien in Deutschland bereits umgesetzt sind und wo noch Defizite bestehen. Dafür wird das HR Konzept kurz eingeführt und es werden die negativen Folgen für Konsumierende und die Gesellschaft umrissen.

\section{Was ist Harm Reduction?}

Die Zielgruppe von HR Strategien umfasst alle Menschen, die sich bewusst für den fortgesetzten Konsum von einer oder mehrerer
Substanz(en) entscheiden oder die aufgrund starker Abhängigkeit sich nicht in der Lage sehen, ihren Konsum einzustellen [18]. Eine breiter gefasste Definition umschließt alle „Interventionen, Programme und Strategien, die auf die Reduzierung der gesundheitlichen, sozialen und ökonomischen Schäden für Individuen und die Gesellschaft durch Substanzkonsum ausgerichtet sind“ (frei übersetzt aus Seite 19: [21]). Damit kann HR sowohl als Ziel effektiver Drogenpolitik verstanden werden, als auch explizite Maßnahmen beschreiben, welche Prävention und Behandlung erweitern, jedoch nicht ersetzen sollen. Das oberste Ziel von HR Programmen ist die Reduzierung der mit dem Konsum verbunden individuellen und gesellschaftlichen Risiken. Für die Umsetzung dieses Ziels ist die Akzeptanz der individuellen Konsumentscheidung eine wichtige Voraussetzung.

„Harm Reduction“ umfasst Maßnahmen, die auf die Reduzierung von möglichen Schäden durch Substanzkonsum ausgerichtet sind.

Während Maßnahmen zur Schadensminderung bereits in den 1920er Jahren entwickelt wurden, erlangte der HR Begriff erst in den 1980er Jahren eine gewissen Bekanntheit, als sich HIV-Infektionen unter Menschen mit injizierendem Heroinkonsum rasant ausbreitete, welche schließlich durch die Bereitstellung sauberer Spritzen eingedämmt werden konnte [22]. Bezüglich des Konsums von Heroin und anderer Opioide bestehen weitere HR Strategien, wie bspw. Substitutionsangebote [23] und Medikamente zur Reduzierung der Todesfälle durch Überdosierung [24-26]. Während HR traditionell v. a. in Bezug auf Risiken durch Opioidkonsum verstanden wurde, sind in den letzten Jahren auch Strategien zur Reduzierung der Risiken von Alkohol- [27], Tabak- [28] und Cannabiskonsum [29] entwickelt worden.

\section{Negative Folgen für Konsumierende und die Gesellschaft}

Da HR Strategien auf die Vermeidung und Reduzierung des potenziellen Schadens abzielen, ist die Kenntnis der cannabisbezogenen Risiken notwendig (für einen systematischen Überblick, siehe [46]). Diese sollen im Folgenden kurz dargestellt werden. Die wichtigste vermeidbare negative Auswirkung des Cannabiskonsums bezieht sich zweifelsohne auf die Entwicklung behandlungsbedürftiger Cannabiskonsumstörungen [30,31]. Weitere Risiken des Cannabiskonsums können sich ergeben, wenn am Straßenverkehr unter akutem Cannabisrausch teilgenommen [32, 33] oder während der Schwangerschaft konsumiert wird [34]. Außerdem finden sich Hinweise für die negativen Auswirkungen des Cannabiskonsums auf die Entwicklung von Schizophrenie und anderer psychischer Probleme [35-37], auf die kognitive Entwicklung im Jugendalter [38, 39], auf die Atemwegsysteme [40, 41] sowie auf das kardiovaskuläre System [42]. Da für diese Problembereiche die kausale Rolle von Cannabiskonsum nicht abschließend geklärt ist, sollten diese Befunde vorsichtig interpretiert werden. 
>Tab. 1 Empfehlungen aus den „Lower-Risk Cannabis Use Guidelines (LRCUG): A Comprehensive Update of Evidence and Recommendations“ [55].

1 Abstinenz ist die beste Möglichkeit Risiken zu vermeiden. Ein fortgesetzter Konsum sollte berücksichtigen, dass die Risikowahrscheinlichkeit und ihr Schweregrad von individuellen Umständen, Konsummustern und der Qualität des konsumierten Produktes abhängen und dass Risiken zwischen Konsumierende und Konsumepisoden schwanken können.

2 Je später junge Menschen mit dem Konsum von Cannabis anfangen, desto geringer ist das Risiko für Einschränkungen in Gesundheit und Wohlergehen im weiteren Lebensverlauf.

3 Konsumierende sollten die Zusammensetzung der psychoaktiven Inhaltsstoffe von Cannabisprodukten kennen. Produkte mit geringen THCGehalt und hohem Verhältnis von Cannabidiol zu THC sollten bevorzugt konsumiert werden.

4 Der Konsum von Produkten mit synthetischen Cannabinoiden sollten vermieden werden.

5 Das Rauchen von Cannabis sollte vermieden und durch weniger schädliche Konsumtechniken wie oralem Konsum und Verdampfen ersetzt werden. Durch oralen Konsum besteht kein Risiko von Atemwegsschädigungen, jedoch können negative Folgen durch nicht-beabsichtigte Einnahme höherer Dosen entstehen, wenn das verzögerte Einsetzen der Wirkung nicht berücksichtigt wird.

6 Beim Rauchen von Cannabis sollte darauf verzichtet werden, den Rauch besonders tief einzuatmen oder ihn verlängert in der Lunge zu behalten. Des Weiteren ist auch der Druckausgleich mittels Ausatmen bei geschlossenen Nasen und Mundöffnungen (auch bekannt als ,Valsalva-Manöver') nach dem Einatmen des Rauches zu vermeiden.

7 Häufiger Konsum (täglich oder fast-täglich) geht einher mit einem erhöhten Risiko für das Erleben gesundheitlicher und sozialer Folgen. Es sollte daher angestrebt werden, den eigenen Konsum und den von Freunden und Freundinnen auf wenige Gelegenheiten (z. B. nur an einem Tag pro Woche, nur am Wochenende) zu begrenzen.

8 Nach dem Konsum von Cannabis sollte mind. 6 Stunden vor dem Führen eines Fahrzeugs (Maschinen, Werkzeuge) gewartet werden. Eine längere Wartezeit kann jedoch bei bestimmten Konsumierenden und spezifischen Cannabisprodukten notwendig sein. Laut der gegenwärtigen Gesetzgebung sollten Konsumierende in Deutschland gänzlich auf das Führen eines Fahrzeuges verzichten.

Des Weiteren besteht ein bedeutend höheres Unfallrisiko nach dem Mischkonsum von Cannabis und Alkohol, weshalb in dieser Situation das Führen eines Fahrzeugs grundsätzlich zu vermeiden ist.

9 Bei Personen mit einer eigenen Neigung zu Psychosen und Substanzkonsumstörungen, Personen bei denen Psychosen oder Substanzkonsumstörungen im ersten Verwandtschaftsgrad auftraten und bei Schwangeren bestehen erhöhte Risiken für negative Folgen von Cannabiskonsum. Diese Personengruppen sollten grundsätzlich kein Cannabis konsumieren.

Um Konsumierende vor möglichen Risiken zu schützen, sollten alle Hinweise auf negative Auswirkungen ernst genommen und möglichst in HR Ansätzen berücksichtigt werden. Dafür lassen sich aus der Forschung Praktiken und Kontextfaktoren des Cannabiskonsums identifizieren, die ausschlaggebend für die entsprechende Risikoexposition sind. Hierbei ist v. a. ein früher Erstkonsum zu nennen, welcher die kognitive Entwicklung im Jugendalter beeinträchtigt $[38,39]$ und das Risiko für Cannabiskonsumstörungen zu erhöhen scheint [43], wobei letzteres vermutlich auch mit der Frequenz des Konsums steigt [44]. Hinweise für Dosis-Wirkungsbeziehungen wurden außerdem für psychotische Symptome [37], Lungenkrebs [45], für Mortalität unter Personen mit Myokardinfarkt [46], für das Unfallrisiko [47], für kognitive Leistungen [48], als auch für Arbeitslosigkeit und Bedarf an Sozialhilfe [49] gefunden. Aus dem gegenwärtigen Stand der Forschung sind zudem bestimmte Risikogruppen identifizierbar, denen jeglicher Cannabiskonsum abzuraten ist. Dazu gehören Menschen mit einer genetischen Prädisposition für psychotische Störungen [36], mit koronaren Herzkrankheiten [42] sowie Schwangere [34].

Die negativen Folgen des Cannabiskonsums lassen sich weiterhin über ihre Prävalenz und Auswirkungen auf Funktionsniveau und Mortalität der Betroffenen als gesamtgesellschaftlicher Schaden quantifizieren. Leider beschränken sich entsprechende Schätzungen auf wenige Studien. Globale [50] und kanadische Schätzungen [8] zeigen, dass ein beträchtlicher Teil der cannabisbezogenen Krankheitslast auf Cannabiskonsumstörungen zurückzuführen ist. Verkehrsunfälle und Lungenkrebs führen zudem zu erhöhter Morbidität und Mortalität und sind damit nicht zu vernachlässigende Faktoren für die gesellschaftliche Belastung $[8,51]$. Für Deutschland liegt zudem eine Schätzung der ökonomischen Belastung der hiesigen Krankenkassen durch Personen mit ICD-10 F12 Diagnosen vor [7], welche sich auf ca. 975 Mio. $€$ pro Jahr summiert.

\section{Aktueller Forschungsstand zu Cannabis und HR}

Potenzielle HR Strategien zur Reduzierung cannabisbezogener Schäden sind vielfältig. Im Folgenden werden bekannte Ansätze auf individueller und struktureller Ebene dargestellt.

\section{Individuelle Ansätze}

Individuelle Ansätze zielen mittels Informationen zum sicheren Umgang mit Cannabis auf die Verantwortung der Konsumierenden. Durch die Einhaltung sogenannter „Safer Use“ Praktiken sollen so die Konsumrisiken gemindert werden. Während in verschiedenen Ländern offizielle Empfehlungen zur Einhaltung bestimmter Trinkmengen [52] sowie für den sicheren Umgang mit verschreibungspflichtigen Medikamenten [53] bestehen, mangelt es an Regeln für einen sicheren Cannabiskonsum. Die bislang umfangreichste Arbeit zu diesem Thema sind die „Lower Risk Cannabis Use Guidelines“ (LRCUG [54, 55]), welche bekannte Risiken zusammenfasst und pragmatische Empfehlungen für Konsumierende ableitet ( Tab. 1).

Die Empfehlungen der LRCUG decken sich weitgehend mit anderen Publikationen zu diesem Thema [56-58]. Im Vergleich zu Richtlinien risikoarmen Alkoholkonsums [59] ist der Bezug auf Konsumquantitäten in den Empfehlungen der LRCUG nicht enthalten. 
Dies lässt sich auf das Fehlen standardisierter Konsumeinheiten zurückführen, welche sowohl die Erforschung von Dosis-Wirkungsbeziehungen als auch die Vermittlung von Grenzwerten für Konsumierende erschwert. Erste Versuche, ein standardisiertes Maß zu entwickeln, könnte jedoch die Differenzierung riskanten Konsumverhaltens mittels Quantität und Frequenz in Zukunft ermöglichen [60-62].

Die „Lower Risk Cannabis Use Guidelines“ zählen zu den individuellen HR Ansätzen - sie enthalten 9 Empfehlungen, bei dessen Einhaltung die Risiken von Cannabiskonsum reduziert werden können.

Die derzeitige Fassung der LRCUG kann außerdem um einige Punkte erweitert werden. Während Punkt 8 die Einhaltung von mind. 6 Stunden Pufferzeit zwischen Konsum und Fahrzeugführung angibt, sollte diese Zeit als absolutes Minimum betrachtet werden. Da die akute Beeinträchtigung durchaus länger anhalten kann, sollten Konsumierende mind. bis zum vollständigen Abklingen des Rausches, möglichst jedoch 24 Stunden warten. Zudem sollte die Beeinträchtigung der Gedächtnisleistung von Konsumierenden berücksichtigt werden $[38,39]$, weshalb der Konsum während Lernphasen vermieden werden sollte.

Weitere Empfehlungen für die Vermeidung von Risiken im Zusammenhang mit dem medizinischen Gebrauch von Cannabisprodukten gibt Grotenhermen [57]. In Übereinstimmung mit den LRCUG wird der orale Konsum empfohlen um die Belastung der Atemwege zu vermeiden. Konsumierende sollten sich an ihre individuell präferierte Dosis vorsichtig mit steigernden Dosen annähern, da das (akute) Risiko oralen Konsums v. a. in einer Überdosierung liegt. Für eine spätere Steigerung der gewünschten Wirkung bieten sich Inhalationstechniken an. Empfehlungen für inhalierenden Konsum beziehen sich unter anderem auf die Verwendung reiner Cannabisprodukte mit höherem $\Delta$ 9-Tetrahydrocannabinol (THC) Gehalt, da mit steigendem Wirkstoffgehaltgehalt insgesamt weniger inhaliert werden muss und damit auch der Beikonsum schädlicher Verbrennungsprodukte wie Teer oder Kohlenmonoxid verringert werden kann. Da derzeit jedoch vermutet wird, dass der THC-Gehalt mit der Auftretenswahrscheinlichkeit psychotischer Symptome assoziiert ist $[63,64]$, ist eine gewisse Vorsicht gegenüber dieser Empfehlung angebracht. Weitere Empfehlungen für die Inhalation von Cannabisprodukten basieren auf dem Vergleich vom Teeranteil im Rauch; demnach ist das Rauchen durch Pfeifen dem mittels joints vorzuziehen, letztere sind jedoch noch risikoärmer als die Verwendung von Wasserpfeifen. Verdampfer werden gemeinhin als schadensärmste Form der Cannabisinhalierung betrachtet [65-69], jedoch wird das Potenzial der Schadensminderung durch Verdampfen im Vergleich zum Rauchen eher als gering eingeschätzt [70]. Es wird auch davor gewarnt, dass mit der Verbreitung von Verdampfern der Konsum von Cannabis generell als risikoärmer wahrgenommen werden könnte, welches einen früheren Erstkonsum begünstigen und die Konsumprävalenz insgesamt erhöhen könnte [71]. Jedoch kann der gesamtgesellschaftliche Effekt dieser beiden entgegenlaufenden Effekte aufgrund mangelnder Studien bislang nicht bewertet werden [72].
Neben Risiken von singulärem Cannabiskonsum sollte besondere Vorsicht vor dem Mischkonsum mit anderen Substanzen bestehen. Da sich beim Konsum verschiedener psychoaktiver Substanzen Wirkungen überlagern und verstärken können, ist anzunehmen, dass das akute Risiko von Kontrollverlust durch die Kombination verschiedener Substanzen ansteigt. Damit verbunden sind - wie in den LRCUG angeführt - erhöhte Unfallrisiken [73] und andere riskante Verhaltensweisen wie ungeschützter Geschlechtsverkehr. Die Kombination von Cannabis mit anderen Substanzen sollte daher nur von erfahrenen Konsumierenden mit entsprechender Vorsicht praktiziert werden. Weiterhin sollte bei wahrgenommenem Kontrollverlust professionelle Hilfe aufgesucht werden. Für die Einschätzung eigener cannabisbezogener Probleme kann die Kenntnis diagnostischer Kriterien für Cannabiskonsumstörungen [30, 31] hilfreich sein. Um eigene Konsummuster zu systematisieren und das damit verbundene Risiko bestimmen zu können, können Konsumierende zudem auf kurze Fragebögen zurückgreifen [74], welche z. T. auch im Internet ausgefüllt werden können (für deutschsprachige Konsumierende, z. B. www.drugcom.de).

Die aufgeführten Empfehlungen zur Schadensminderung können auf unterschiedliche Art und Weise eingebettet werden. Jedoch erscheint die Verbreitung dieser Informationen unter jungen Menschen besonders wichtig, da diese den Umgang mit Cannabis noch erlernen und ein großer Teil cannabisbezogener Schäden erst nach einigen Jahren Konsum entsteht und daher noch vermieden werden kann. Akteure der Suchtprävention, d. h. Beratungsstellen, Jugendclubs, Schulen und Universitäten, könnten hierbei eine zentrale Rolle spielen, da die Präventionsarbeit mit Jugendlichen entsprechendes Wissen erhöhen kann und Konsumabsichten hinterfragen, aber auch den Konsum selber reduzieren kann [75-79]. Studien zur Evaluierung von Suchtprävention mit expliziten HR Merkmalen sind bislang eher selten, aber erfolgversprechend [80-82]. Die Implementierung von HR in der Drogenaufklärung unter Jugendlichen muss jedoch verstärkt darauf achten, wie Hinweise zur Schadensminderung an die Zielgruppe übermittelt werden, da durch Aufklärung der Substanzkonsum potenziell auch ansteigen kann [83]. Dementsprechend sind eine angemessene Sprache, die Auswahl der richtigen (medialen) Kanäle, die Anpassung der Inhalte an Merkmale der Zielgruppe, wie z. B. Alter [84] wichtig, um zu vermeiden, dass Cannabiskonsum durch HR Strategien verharmlost wird [56]. Leider wird die Implementierung effektiver Interventionsansätze in der Drogenaufklärung durch die starke Ideologisierung erschwert [85], die sich im Spannungsfeld zwischen einer prohibitorischen Perspektive einerseits und liberalen Ansätzen andererseits, die die eigenständige und informierte Entscheidung der Jugendlichen stärken, befindet [86, 87].

Neben der Suchtprävention könnte ein risikoarmer Cannabiskonsum auch in sogenannten Kurzinterventionen thematisiert werden. Hierbei werden in kurzen Gesprächen problematische Konsummuster adressiert und Handlungsempfehlungen können zu einer Reduzierung risikoreicher Konsumpraktiken führen und bieten daher grundsätzlich ein großes Potenzial (z. B. [88]). Es besteht hierbei jedoch eine große Überschneidung zur Behandlung von cannabisbezogenen Störungen, da Kurzinterventionen nur für eine begrenzte Population als sinnvoll erachtet werden und Menschen mit hohem Konsum und/oder entsprechenden Schädigungen in die spezialisierte Versorgung überwiesen werden sollten [89]. Kur- 
zinterventionen für problematischen Alkoholkonsum zielen üblicherweise auf die Reduzierung von Trinkmengen ab. Da sie gut erforscht sind [90] und robuste Wirksamkeitshinweise vorliegen [91], werden sie als eine zentrale Methode zur Reduzierung globaler alkoholbezogener Schäden empfohlen [92]. Kurzinterventionen für Cannabis zielen ebenso auf die Reduzierung des Konsums oder auf die Einhaltung von Abstinenz ab, indem sie sich klassischer Elemente der kognitiv-behavioralen Verhaltenstherapie, wie dem „Motivational Interviewing“ bedienen (siehe z. B. [93, 94]. Denkbar ist die Anwendung dieser Kurzinterventionen bei jungen Patienten und Patientinnen (15-30-jährigen) in der allgemeinärztlichen Versorgung [95]. Da Cannabiskonsum in dieser Altersgruppe besonders verbreitet ist, könnte ein regelmäßiges Screening begleitet durch Empfehlungen für einen sicheren Konsum eine sinnvolle HR Maßnahme sein. Regelmäßige Screenings können außerdem dazu beitragen, problematische Konsummuster frühzeitig zu erkennen und die Betroffenen an entsprechende Behandlungsangebote zu verweisen. Es mangelt jedoch derzeit an Studien zu Kurzinterventionen mit expliziten HR Ansätzen, sodass keine Aussagen über deren Wirksamkeit getätigt werden können.

\section{Strukturelle Ansätze}

Ansätze auf struktureller Ebene gehen über den individuellen Konsum hinaus und versuchen vielmehr das Umfeld der Konsumierenden so zu gestalten, dass Schaden vermieden oder reduziert werden kann. Aus der Erfahrung mit anderen Substanzen zeigt sich bspw., dass der Zugang zu sicheren Konsumräumen die Gesundheit von Menschen mit injizierendem Drogenkonsum erhöht und Risiken, wie z. B. Überdosen und Infektionsraten, reduziert hat [96]. Diese Erfahrung lässt sich im weiteren Sinne auf Cannabis übertragen, da bestimmte Risiken, die mit dem Konsum von Cannabis einhergehen, durch die Umgebung modifiziert werden können. Hier ist insbesondere der Wirkstoffgehalt und Reinheitsgrad von Cannabis zu nennen. Da Cannabidiol unerwünschte Effekte von THC zu modulieren scheint $[55,97,98]$, ist die Kenntnis der wirksamen Inhaltsstoffe und deren Stärke daher eine wichtige Voraussetzung, um die optimale individuelle Dosis bestimmen zu können.

Außerdem sollte der Beikonsum schädlicher Nebenprodukte vermieden werden. Die Bestimmung von Verunreinigungen ist wichtig, da Cannabis mit Pestiziden, Blei, Plastik und anderen teils giftigen Substanzen belastet sein kann. Zur Bestimmung des Wirkstoffgehaltes und möglicher Belastungen sollten Cannabisprodukte entweder staatlich oder durch interessierte Konsumierende selber überprüft werden können. Im Sinne der bestmöglichen Schadensreduzierung wäre eine flächendeckende Kontrolle von Cannabisprodukten - analog zu anderen (legalen) Rauschmitteln - hinsichtlich Reinheitsgrad und Wirkstoffgehalt wünschenswert. Eine staatliche Qualitätskontrolle bedarf jedoch einer legalen Abgabe und strikten Regulierung von Cannabis [99], wie bspw. derzeit in Kanada angestrebt [100,101]. In Regionen ohne zentrale Kontrollinstanz bestünde die Möglichkeit, dass lokale „drug checking“ Initiativen diese Rolle z. T. übernehmen. Konsumierende könnten bei diesen Initiativen Substanzproben abgeben und auf entsprechende Parameter untersuchen lassen. Europäische „drug checking“ - Initiativen ermöglichen das Testen illegaler Substanzen auf Partys und Festivals („on-site“) oder in stationären Laboren. Wissenschaftliche Untersuchungen zu HR Aspekten von „drug checking " sind bislang rar, weisen jedoch darauf hin, dass die Beschäftigung mit Wirkstoffgehalt und möglichen Verunreinigungen eine Reflektion des eigenen Konsums anzuregen scheint. Es kann aber auch nicht ausgeschlossen werden, dass manche Menschen einen Anreiz darin sehen, entsprechende Substanzen zu konsumieren oder einen bereits intendierten Konsum zu intensivieren [102]. Es fehlt allerdings eine systematische Untersuchung der möglichen Kosten und Nutzen von „drug checking“ - Angeboten, um diese konträren Effekte gegeneinander abwägen zu können.

Derzeit fokussieren „drug checking“ Angebote zumeist auf andere illegale Drogen [102-105], jedoch ist es denkbar diese Ansätze auch für Cannabis zu erweitern. Institutionen, die „drug checking“ - anbieten, sind zudem prädestiniert für die Verbreitung von HR Informationen, da diese Angebote verstärkt von Menschen mit hochfrequentem und dementsprechend risikoreichem Konsum genutzt werden [106]. Neben dem direkten Kontakt, welcher von Aufklärungsarbeit und Unterstützung bis hin zu Therapievermittlung reichen kann, ermöglichen „drug checking“ Strukturen einerseits ein kontinuierliches Monitoring des konsumierten Cannabis um regionale Trends im Wirkstoffgehalt und Verunreinigungen zu identifizieren und andererseits können diese Informationen schnell unter Konsumierenden verbreitet werden $[102,105]$. Erfahrungen mit „drug checking“ in Bezug auf Cannabis sind jedoch sehr rar (Monitoring von Cannabisprodukten in den Niederlanden: $[107,108]$ ) und das Potenzial zur Schadensminderung ist bislang kaum erforscht.

Während viele Maßnahmen unabhängig von der legalen Regulierung des Besitzes und Konsums von Cannabis implementiert werden und wirken können, sollten der rechtliche Umgang und seine Auswirkungen explizit aus der Perspektive der Schadensminderung beleuchtet werden. So sind durch die Prohibition von Cannabisbesitz und -handel hohe ökonomische Kosten zu beziffern, die auf Strafverfolgung und entgangene Steuereinnahmen zurückgehen [109]. Die individuellen Folgen für Konsumierende spiegeln sich dabei in der Strafverfolgung, Stigma, Arbeitsplatzverlust und Führerscheinentzug wider [58, 99, 110-112]. Eine Entpönalisierung, Entkriminalisierung oder Legalisierung könnte den entsprechenden Schaden reduzieren [58]. Jedoch ist hier eine genaue Abwägung der Vor- und Nachteile entsprechender Regulationsmöglichkeiten vonnöten $[99,110,113,114]$.

\section{Cannabis und HR in Deutschland}

Die offizielle Drogen- und Suchtstrategie der Bundesregierung aus dem Jahr 2012 begriff HR als 1 von 4 Ebenen, neben Prävention, Behandlung und Repression, jedoch fehlte hier bereits ein konkreter Bezug schadensmindernder Maßnahmen für Cannabis [115]. Mit Annahme der Gesamteuropäischen Drogenstrategie wurde HR dem Ziel der Nachfragereduzierung untergliedert [116]. In anderen Worten wurde Schadensminimierung damit der Status als eigenständiges drogenpolitisches Ziel aberkannt und es wurde stattdessen zu einem Mittel zur Reduzierung der Drogennachfrage erklärt. Explizit werden Todesfälle und Infektionen, die auf Drogenkonsum zurückgehen, genannt, welche durch HR Maßnahmen zu vermeiden sind. Mögliche Maßnahmen zur Reduzierung cannabisbezogener Schäden fehlen hier völlig. 


\section{Umsetzung individueller Ansätze}

Die mangelhafte Berücksichtigung von HR in nationalen und europäischen Drogenstrategien spiegeln sich auch in der offiziellen Informationspolitik bezüglich Cannabis in Deutschland wider. Auf der Webseite der Bundeszentrale für gesundheitliche Aufklärung werden Informationen bezüglich Cannabis v. a. im Sinne einer Primärprävention angeboten, so z. B. in einer Broschüre der Deutschen Hauptstelle für Suchtfragen, die allerdings keine Informationen für einen sicheren Gebrauch von Cannabis enthält [117]. Konsumierende, die nach deutschsprachigen Informationen für einen sicheren Gebrauch von Cannabis suchen, finden diese eher bei lokalen Initiativen wie dem Therapieladen e.V. (z. B. Broschüre: „Cannabis denn Sünde sein“ [118]), chill out e.V. (http://chillout-pdm. de/substanzinfos/cannabis/), Alice-Project (http://www.alice-project.de/) oder bei den Drug Scouts (https://drugscouts.de/de/lexikon/cannabis), welche oftmals als Vorreiter für HR Ansätze gelten [119]. Umfangreichere Informationen bieten dagegen offizielle Stellen in anderen Ländern (z. B. Colorado (USA): [https://www. colorado.gov/pacific/marijuana/responsible-use], Washington (USA): [http://learnaboutmarijuanawa.org/], Vancouver (Kanada): [http://www.vch.ca/media/TakeCarewithCannabis.pdf/] und Australien [https://ncpic.org.au/]).

Die Informationsvermittlung im Rahmen der Suchtprävention an deutschen Schulen ist durch das föderale System nicht einheitlich geregelt und kaum Bestandteil wissenschaftlicher Evaluationen, wobei bestehende verhaltenspräventive Ansätze neben Verzögerung des Erstkonsums und genereller Verhinderung des Konsums auch auf die Reduzierung schädlicher Konsumfolgen abzielen [120]. Es mangelt jedoch an systematischen Studien um die tatsächliche Praxis der Suchtprävention an deutschen Schulen genauer beschreiben zu können. Im Sinne der offiziellen Informationspolitik ist aber anzunehmen, dass HR Konzepte im Alltag lediglich auf den Umgang mit Alkohol und kaum auf andere Substanzen angewendet werden. Dementsprechend kann vermutet werden, dass ein sicherer Umgang von Cannabiskonsum in deutschen Schulen nicht thematisiert wird, sondern Abstinenz als oberstes oder ausschließliches Ziel ausgegeben wird.

Da durch eine Abstinenzfokussierung der Cannabiskonsum grundsätzlich als defizitär, sowie beratungs- oder behandlungsbedürftig eingeordnet wird, besteht die Gefahr, dass Jugendliche mit Drogenerfahrungen stigmatisiert werden [121]. Zudem ist fraglich, ob Abstinenz als ein realistisches Ziel von Jugendlichen akzeptiert werden kann, da fast jeder fünfte Schüler und jede achte Schülerin angibt im letzten Jahr Cannabis konsumiert zu haben [122] und auch der Erstkonsum von Cannabis hauptsächlich im Schulalter zu verorten ist (d. h. zwischen 14 und 19 Jahren, [123]). Alternativ zur abstinenzorientierten Suchtprävention wurde eine akzeptierende Drogenerziehung vorgeschlagen, die darauf abzielt, Jugendlichen unter Berücksichtigung der Vermeidung negativer Konsumaspekte einen mündigen Umgang mit Drogenkonsum zu vermitteln [121]. Auch wenn dieser Vorschlag sich bislang nicht durchgesetzt hat, reiht er sich ein in die geschichtliche Entwicklung der Suchtprävention, die sich zusehends von der Abstinenzorientierung löst und stattdessen Individuen in ihren souveränen Entscheidungen und Selbstkontrollbemühungen unterstützt [124]. Diese Tendenz kann auch durch die kürzlich veröffentlichten Materialien zur Förderung der Drogenmündigkeit [125] bestärkt und vorangetrieben werden. Weitere Aufklärungskonzepte können auBerdem durch die Identifikation effektiver Elemente in der Suchtprävention entwickelt werden [126], da hierzu bereits umfangreiche Erkenntnisse aus anderen Ländern vorliegen [75, 77]. Aspekte zur Schadensminderung sollten hierbei beachten werden, wurden bislang aber wenig erforscht $[80,127]$. Wichtig bleibt jedoch zu beachten, dass die Thematisierung von sicherem Cannabiskonsum im Jugendalter keinen zusätzlichen Anreiz zur Initiierung von Cannabiskonsum bieten soll/darf. Zudem besteht die Gefahr, dass eine solche Aufklärung unter Umständen als "Verharmlosung” und damit als indirekte Konsumaufforderung interpretiert wird, welches wiederum eine Straftat darstellt. Maßgebendes Ziel sollte daher sein, den Erstkonsum bis zum Erwachsenenalter zu verzögern und bereits konsumierende Jugendliche darin zu bestärken, einen verantwortungsvollen Umgang mit Cannabis zu erlernen.

Während die Implementierung von HR Konzepten im Rahmen der Suchtprävention bislang mangelhaft ist, kann in der Suchthilfe ein grundsätzlich positiver Trend beobachtet werden [12]. So wird in der aktuell gültigen AWMF Leitlinie zur Behandlung von cannabisbezogenen Störungen nach dem Behandlungsbedarf von unterschiedlichen diagnostischen Gruppen (z. B. Intoxikation, Entzug, Abhängigkeit, Flashback, Komorbidität, usw.) differenziert. Das primäre Ziel ist gemäß der Leitlinie nicht Abstinenz, sondern die „Behandlung und Vermeidung von Intoxikationen und schädlichem Gebrauch“ ([128], Seite 325). Der größte Anteil der rund 32000 Konsumierenden, die im Suchthilfesystem erfasst wurden [129], wird ambulant versorgt [130]. In diesem Bereich wurden verschiedene niedrigschwellige Angebote entwickelt, die auf individuelle Konsummuster und -entscheidungen eingehen. Diese zielen mittels Beratung und Gruppensitzungen darauf ab den Konsum zu reflektieren [131], ihn zu reduzieren oder ganz einzustellen [132]. Zudem bestehen Online-Angebote, welche dabei unterstützen den eigenen Konsum selbst einzuschätzen (https://www.drugcom. de/?id = cannabischeck) oder zu reduzieren [133]. Die Entwicklung der erwähnten Modellprojekte soll positiv hervorgehoben werden, jedoch ist nicht zu beurteilen, ob und inwieweit sich der Behandlungsalltag dadurch verändert hat [12].

\section{Umsetzung struktureller Ansätze}

Entsprechend der beschriebenen Drogenstrategien fehlen in Deutschland auch strukuturelle HR Maßnahmen bezüglich Cannabis. Während in anderen europäischen Ländern „drug checking“ von nicht staatlichen oder staatlichen Institutionen durchgeführt wird, wird die Analyse des Wirkstoffgehalts illegaler Substanzen in Deutschland nur Pharmazeuten und Behörden zugestanden [102, 104]. Durch Ausnahmeregelungen konnten lokale Initiativen für begrenzte Zeiträume „drug checking“ anbieten, jedoch wurden Vorschläge, dies hierzulande nach dem Vorbild anderer europäischer Staaten zu ermöglichen, nicht stattgegeben [102,134]. Dementsprechend kann das Testen von Cannabisproben auf Wirkstoff und Reinheit in Deutschland zurzeit lediglich privat mittels frei erwerblicher Geräte und Utensilien (z. B. Teststreifen) durchgeführt werden. Informationen zu gestreckten Cannabisprodukten können zudem über freiwillige Meldungen bezogen werden (z. B. über http://www.streckmittelmelder.de/). Diese Angaben sind jedoch nicht gesichert und umfassen keine Angaben zum Wirkstoffgehalt. 
Auf der legalen Ebene sind die Gesetzgebungen zu Erwerb, Besitz und Konsum von Cannabis und Teilnahme am Straßenverkehr unter Substanzeinfluss zu unterscheiden: Laut $\$ 29$ des Betäubungsmittelgesetzes (BtMG) kann der Besitz von Cannabis mit bis zu 5 Jahren Freiheitsentzug oder einer entsprechenden Geldstrafe bestraft werden. Im Jahr 2016 wurden 145915 Fälle im Zusammenhang mit dem Verstoß gemäß BtMG $\$ 29$ mit Cannabis gezählt [135], wobei in den letzten Jahren ein problematischer Anstieg der Repression v. a. in Bezug auf junge Konsumierende zu beobachten ist [136]. In Deutschland werden grundsätzlich alle Verstöße gemeldet, jedoch können Staatsanwaltschaft oder Gericht von der Strafverfolgung absehen, sofern es sich um eine sogenannte geringe Menge zum Eigenverbrauch handelt (BtMG §31a). Da die Grenzwerte zur geringen Menge und die Verfahren zur Einstellung der Strafverfolgung nicht eindeutig geregelt sind (Unterschiede von Bundesland zu Bundesland und von Fall zu Fall) [1, 137], können sich Konsumierende nicht darauf verlassen, dass ein Cannabisdelikt schließlich nicht verfolgt wird. Der relativ hohe Anteil eingestellter Verfahren [137] kann aber als Indikator für die Vermeidung unnötiger Folgeschäden durch Strafverfolgung betrachtet werden.

Im Gegensatz dazu besteht im Führerscheinrecht kein äquivalenter Handlungsspielraum. Wem der Konsum von Cannabis beim Führen eines Fahrzeugs nachgewiesen wird, kann mit einer Freiheitsstrafe von bis zu einem Jahr oder einer äquivalenten Geldstrafe bestraft werden. Grundsätzlich kann so jeglicher Konsum zum Entzug des Führerscheins führen [138], jedoch gilt ein THC-Gehalt von $1 \mathrm{ng} / \mathrm{ml}$ Blut als unterer Grenzwert, äquivalent zum 0,5\% Wert für Alkoholkonsum. Dieser ist allerdings nicht gesetzlich festgelegt, sondern basiert auf verschiedenen Rechtsprechungen [1]. Außerdem repräsentiert er im Vergleich mit anderen europäischen Ländern und den USA eher das untere Ende implementierter Grenzwerte [139]. Laut Grotenhermen et al. [140] läge ein vergleichbarer Grenzwert zur 0,5\% Grenze für Alkohol eher bei 3,5-5 ng/ml Blut. Demnach ist davon auszugehen, dass es in Deutschland eine nicht zu vernachlässigende Anzahl Personen gibt, die von den negativen Folgen der Rechtsprechung (Führerscheinentzug und Folgeschäden, wie z. B. Arbeitsplatzverlust) betroffen sind, obwohl sie ohne akute Beeinträchtigung am Straßenverkehr teilgenommen haben [139] oder weil die zuständige Führerscheinbehörde durch eine andere Behörde im Zusammenhang mit einem anderen Cannabisdelikts informiert wurde [138].

\section{Zusammenfassung und Implikationen}

Diese Arbeit fasst den gegenwärtigen Forschungsstand von HR Strategien in Bezug auf Cannabis zusammen und illustriert, in welchem Umfang Strategien zur Reduzierung und Vermeidung von cannabisbezogenem Schäden in Deutschland angewendet werden.

Es bestehen zahlreiche Möglichkeiten, die darauf abzielen, potenzielle Risiken und tatsächliche Schäden von Konsumierenden zu reduzieren oder zu vermeiden. Die beschriebenen Strategien, Empfehlungen und Programme entstammen größtenteils der Forschung oder lokaler Initiativen und spiegeln sich kaum in der offiziellen Drogenpolitik der Bundesregierung wider. Obwohl HR als 1 der 4 Pfeiler deutscher Drogenpolitik gilt, sind explizite Anstrengungen zur Reduzierung cannabisbezogener Schäden in Deutschland kaum präsent. Das womöglich größte Potenzial sehen wir darin, einen verantwortungsvollen, risikoarmen Gebrauch von Cannabis überhaupt ansprechen zu können. Während in der ambulanten Suchthilfe diesbezüglich eine positive Entwicklung zu beobachten ist, ist die Implementierung in der Suchtprävention, v. a. in der schulischen Suchtprävention, mangelhaft. Bundesweite (z. B. Bundeszentrale für gesundheitliche Aufklärung) und lokale Akteure (z. B. Beratungsstellen, Jugendtreffs, Schulen und Universitäten) sollten Verantwortung übernehmen und Informationen für einen möglichst risikoarmen Cannabiskonsum anbieten (z. B. als Teil des Informationsmaterial für alle neuen Studierenden). Darüber hinaus sollte auch diskutiert werden, wie effektiv die Suchtprävention an deutschen Schulen derzeit ist und inwieweit die Thematisierung eines mündigen Umgangs mit Cannabis umsetzbar ist.

Entgegen der offiziellen Drogenpolitik und trotz vielfältigen Möglichkeiten fehlen in Deutschland explizite Maßnahmen zur Reduzierung der cannabisbezogenen Schäden.

Obwohl einige Möglichkeiten zur Reduzierung von cannabisbezogenen Schäden identifiziert wurden, können kaum Aussagen über deren Umsetzbarkeit und Wirksamkeit getroffen werden, da bislang nur wenige Studien diesbezüglich durchgeführt wurden. Das könnte damit zu erklären sein, dass der Konsum von Cannabis im Vergleich mit anderen Substanzen vergleichsweise geringe Schäden verursacht $[50,141]$ und deswegen die Anstrengungen zur Reduzierung dieser Schäden entsprechend begrenzt sind. Angesichts des hohen Anteils an Konsumierenden und der vielfältigen Konsumrisiken sind Anstrengungen zur Umsetzung und Evaluation der beschriebenen Strategien durchaus angebracht. Dieser Prozess wird längerfristig notwendig sein um eine evidenzbasierte Drogenpolitik gestalten zu können [142].

Die Beobachtung, dass sich drogenpolitische Strategien bezüglich Cannabis derzeit kaum an HR Prinzipien orientieren, mag als Ausdruck einer prohibitionistischen Ausrichtung interpretiert werden. Die damit verbundene Abstinenzorientierung impliziert, dass jeglicher Konsum von Cannabis schädlich sei und von daher vermieden werden sollte. Daher sind drogenpolitische Strategien traditionell auf die Reduzierung des Konsums und Handels ausgerichtet $[9,110]$. Die deutsche Drogenpolitik ist jedoch nicht ausschließlich von der Ideologie einer radikalen Prohibition geprägt, sondern übernimmt zusehends Public Health- und humanistische Aspekte. So steht laut der nationalen Drogenstrategie „der suchtgefährdete oder von der Sucht betroffene Mensch [...] im Mittelpunkt“ und entsprechende Angebote müssen sich „an der Lebensrealität der Betroffenen orientieren“ (Seite 3, [115]). Dass ein Umdenken in der Drogenpolitik also längst stattgefunden hat, lässt sich auch im jüngsten Vorstoß zur Regulierung des medizinischen Gebrauchs von Cannabis erkennen [143]. In diesem (ideologischen) Rahmen ist die Berücksichtigung schadensreduzierender Strategien durchaus denkbar, welche sich zudem in der Mehrzahl innerhalb der derzeitigen legalen Regulierung integrieren lassen.

Über den politischen Gestaltungsspielraum hinaus sollte zudem der gesellschaftliche Umgang mit Cannabis eine größere Bedeutung zugemessen bekommen. Während für Alkohol distinkte Trinkkulturen identifiziert wurden (für Europa, siehe [144]), sind regional abgrenzbare Cannabiskulturen bislang nicht eindeutig charakterisiert. 
Für eine HR Perspektive wären hierfür relevante Charakteristika unter anderem in der Risikowahrnehmung [145] und im Beikonsum mit Tabak [146] zu finden, da sie vorherrschende Normen prägen [147] und somit die Ausübung und Akzeptanz von riskantem Konsumverhalten beeinflussen.

\section{FAZIT FÜR DIE PRAXIS}

Der Konsum von Cannabis ist in Deutschland weit verbreitet und mit individuellen Risiken und gesellschaftlichem Schaden verbunden. Es bestehen vielfältige Ansätze um Konsumrisiken reduzieren und Schaden vermeiden zu können, die sich z. T. im Rahmen von Prävention, Intervention und Repression widerspiegeln. Eine eigenständige Drogenpolitik mit explizitem HR Charakter ist jedoch nicht vorhanden. Die Übernahme verschiedener Strategien zur Risiko-und Schadensminderung erscheint dagegen vor dem legalen und ideologischen Hintergrund durchaus machbar und wünschenswert und würde die Glaubwürdigkeit und Zielgenauigkeit der cannabisbezogenen Drogenpolitik erhöhen.

\section{Autorinnen/Autoren}

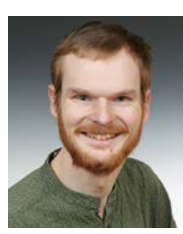

\section{Jakob Manthey}

studierte bis 2013 an der Technischen Universität Dresden Psychologie. In seiner Diplomarbeit evaluierte er die „Lower Risk Cannabis Use Guidelines“ am Centre for Addiction and Mental Health in Toronto, Kanada. Seit seinem Abschluss arbeitet er am Institut für Klinische Psychologie und Psychotherapie an der Technischen Universität Dresden und hat unter anderem eine Studie zur Erforschung der Alkoholabhängigkeit in der allgemeinärztlichen Versorgung koordiniert.

\section{Interessenkonflikt}

Die Autoren geben an, dass keine Interessenkonflikte bestehen.

\section{Literatur}

[1] Pfeiffer-Gerschel T, Jakob L, Dammer E et al. Bericht 2015 des nationalen REITOX-Knotenpunkts an die EBDD. München: DBDD; 2015

[2] Pabst A, Kraus L, Matos EGd et al. Substanzkonsum und substanzbezogene Störungen in Deutschland im Jahr 2012. Sucht 2013; 59: 321-331

[3] Orth B. Die Drogenaffinität Jugendlicher in der Bundesrepublik Deutschland 2015. Rauchen, Alkoholkonsum und Konsum illegaler Drogen: aktuelle Verbreitung und Trends. In, BZgA-Forschungsbericht. Köln: Bundeszentrale für gesundheitliche Aufklärung; 2016

[4] Hall W, Degenhardt L. The adverse health effects of chronic cannabis use. Drug testing and analysis 2013; 6: 39-45

[5] Hoch E, Bonnetn U, Thomasius R et al. Risks associated with the non-medicinal use of cannabis. Deutsches Arzteblatt international 2015; $112: 271-278$
[6] World Health Organization. The health and social effects of nonmedical cannabis use. In: Hall W, Renström M, Poznyak V. eds.Geneva: WHO Press; 2016

[7] Effertz T, Verheyen F, Linder R. Ökonomische und intangible Kosten des Cannabiskonsums in Deutschland. Sucht 2016; 62: 31-41

[8] Imtiaz S, Shield KD, Roerecke M et al. The burden of disease attributable to cannabis use in Canada in 2012. Addiction 2016; 111: 653-662

[9] Simon R. Prohibition, Legalisierung, Dekriminalisierung: Diskussion einer Neugestaltung des Cannabisrechts. Sucht 2016; 62: 43-50

[10] Thomasius R, Holtmann M, Melchers P et al. Für Sie notiert - Cannabispolitik in Deutschland hat sich bewährt. Suchttherapie 2016; 17: 57-59

[11] Ballotta D, Pfeiffer-Gerschel T, Goosdeel A. Is UNGASS 2016 the turning point in international drug policy? Sucht 2016; 62: $59-60$

[12] Tossmann P, Gantner A. Frühintervention, Beratung und Behandlung bei Cannabisstörungen. Suchttherapie 2016; 17: 85-89

[13] European Monitoring Centre for Drugs and Drug Addiction. European Drug Report 2016: Trends and Developments. Luxembourg: Publications Office of the European Union; 2016

[14] Williams J. The effects of price and policy on marijuana use: what can be learned from the Australian experience? Health economics 2004; 13: $123-137$

[15] van Ours JC, Williams J. Cannabis prices and dynamics of cannabis use. J Health Econ 2007; 26: 578-596

[16] Shi Y, Lenzi M, An R. Cannabis liberalization and adolescent cannabis use: A cross-national study in 38 countries. PloS one 2015; 10 : e0143562

[17] Pacula RL, Kilmer B, Wagenaar AC et al. Developing public health regulations for marijuana: Lessons from alcohol and tobacco. American Journal of Public Health 2014; 104: 1021-1028

[18] International Harm Reduction Association. Was ist Harm Reduction? Eine Erklärung der International Harm Reduction Association. (April 2010). Im Internet: https://www.hri.global/files/2010/06/01/ Briefing_What_is_HR_German.pdf; Zugriff: 28.11.2016

[19] Michels II, Stöver H. Harm reduction-from a conceptual framework to practical experience: the example of Germany. Substance use \& misuse 2012; 47: 910-922

[20] Meyer-Thompson H-G, Stöver H. Cannabiskonsum aus dem Blickwinkel von Schadensminderung/harm reduction und Public Health. In: Stöver H, Werse B. (eds). 3 Alternativer Drogen- und Suchtbericht 2016. Lengerich, Germany: Pabst Science Publishers; 2016

[21] European Monitoring Centre for Drugs and Drug Addiction. Harm reduction: evidence, impacts and challenges. In: Rhodes T, Hedrich D. eds.Luxembourg: Publications Office of the European Union; 2010

[22] Cook C, Bridge J, Stimson GV. The diffusion of harm reduction in Europe and beyond. In: Rhodes T, Hedrich D. (eds). Harm reduction: evidence, impacts and challenges. Luxembourg: Publications Office of the European Union; 2010: 37-58

[23] MacArthur G], Minozzi S, Martin N et al. Opiate substitution treatment and HIV transmission in people who inject drugs: systematic review and meta-analysis. Bmj 2012; 345: e5945

[24] Dichtl A, Stöver H, Dettmer K. „Naloxon kann Leben retten!“ - TakeHome-Naloxon-Programme als Prophylaxe tödlicher Drogennotfälle ["Naloxone Can Save Lives!" - Take-Home Naloxone Programs as Prophylaxis of Opiate Overdose Fatalities]. Suchttherapie 2016; 17: 137-143

[25] Beletsky L, Rich JD, Walley AY. Prevention of fatal opioid overdose. JAMA 2012; 308: 1863-1864

[26] World Health Organisation. Community management of opioid overdose. Geneva: WHO Press 2014 
[27] Herring R, Thom B, Beccaria F et al. Alcohol harm reduction in Europe. In: Rhodes T, Hedrich D. (eds). Harm reduction: evidence, impacts and challenges. Luxembourg: Publications Office of the European Union; 2010: 275-302

[28] Gartner C, Hall W, McNeill A. Harm reduction policies for tobacco. In: Rhodes T, Hedrich D. (eds). Harm reduction: evidence, impacts and challenges. Luxembourg: Publications Office of the European Union; 2010: 255-274

[29] Hall W, Fischer B. Harm reduction policies for cannabis. In: Rhodes T, Hedrich D. (eds). Harm reduction: evidence, impacts and challenges. Luxembourg: Publications Office of the European Union; 2010: 235-254

[30] American Psychiatric Association. Diagnostic and Statistical Manual of Mental Disorders. 5. Aufl. Washington, DC: American Psychiatric Association; 2013

[31] World Health Organization. The ICD-10 classification of mental and behavioural disorders: Diagnostic criteria for research. Geneva: World Health Organization; 1993

[32] Li MC, Brady JE, DiMaggio C] et al. Marijuana use and motor vehicle crashes. Epidemiologic reviews 2012; 34: 65-72

[33] Asbridge M, Hayden JA, Cartwright JL. Acute cannabis consumption and motor vehicle collision risk: systematic review of observational studies and meta-analysis. Bmj 2012; 344: e536

[34] Wu C-S, Jew CP, Lu H-C. Lasting impacts of prenatal cannabis exposure and the role of endogenous cannabinoids in the developing brain. Future neurology 2011; 6: 459-480

[35] Moore THM, Zammit S, Lingford-Hughes A et al. Cannabis use and risk of psychotic or affective mental health outcomes: a systematic review. The Lancet 2007; 370: 319-328

[36] Shrivastava A, Johnston M, Terpstra K et al. Pathways to psychosis in cannabis abuse. Clinical Schizophrenia \& Related Psychoses 2015; 9: 30-35

[37] Marconi A, Di Forti M, Lewis CM et al. Meta-analysis of the Association Between the Level of Cannabis Use and Risk of Psychosis. Schizophrenia bulletin 2016, doi:10.1093/schbul/sbw003

[38] Crane NA, Schuster RM, Fusar-Poli P et al. Effects of cannabis on neurocognitive functioning: recent advances, neurodevelopmental influences, and sex differences. Neuropsychology review 2013; 23: $117-137$

[39] Walsh Z, Gonzalez R, Crosby K et al. Medical cannabis and mental health: A guided systematic review. Clin Psychol Rev 2016; 51: 15-29

[40] Joshi M, Joshi A, Bartter T. Marijuana and lung diseases. Current opinion in pulmonary medicine 2014; 20: 173-179

[41] Tashkin DP. Effects of marijuana smoking on the lung. Annals of the American Thoracic Society 2013; 10: 239-247

[42] Franz CA, Frishman WH. Marijuana use and cardiovascular disease. Cardiology in review 2016; 24: 158-162

[43] Butterworth P, Slade T, Degenhardt L. Factors associated with the timing and onset of cannabis use and cannabis use disorder: results from the 2007 Australian National Survey of Mental Health and Well-Being. Drug and alcohol review 2014; 33: 555-564

[44] Noack R, Hofler M, Lueken U. Cannabis use patterns and their association with DSM-IV cannabis dependence and gender. Eur Addict Res 2011; 17: 321-328

[45] Aldington S, Harwood M, Cox B et al. Cannabis use and risk of lung cancer: a case-control study. The European respiratory journal 2008; 31: $280-286$

[46] Mukamal KJ, Maclure M, Muller JE et al. An exploratory prospective study of marijuana use and mortality following acute myocardial infarction. American heart journal 2008; 155: 465-470

[47] Ramaekers JG, Berghaus G, van Laar M et al. Dose related risk of motor vehicle crashes after cannabis use. Drug and alcohol dependence 2004; 73: 109-119
[48] Bolla KI, Brown K, Eldreth D et al. Dose-related neurocognitive effects of marijuana use. Neurology 2002; 59: 1337-1343

[49] Danielsson AK, Falkstedt D, Hemmingsson T et al. Cannabis use among Swedish men in adolescence and the risk of adverse life course outcomes: results from a 20 year-follow-up study. Addiction 2015; 110: 1794-1802

[50] Degenhardt L, Ferrari A], Calabria B et al. The global epidemiology and contribution of cannabis use and dependence to the global burden of disease: results from the GBD 2010 study. PloS one 2013; 8: e76635

[51] Fischer B, Imtiaz S, Rudzinski K et al. Crude estimates of cannabisattributable mortality and morbidity in Canada-implications for public health focused intervention priorities. Journal of public health 2016; 38: $183-188$

[52] Furtwaengler NA, de Visser RO. Lack of international consensus in low-risk drinking guidelines. Drug and alcohol review 2013; 32: 11-18

[53] Wise J. NICE calls for safer use of controlled drugs. Bmj 2016; 353 : i2137

[54] Fischer B, Jeffries V, Hall W et al. Lower Risk Cannabis Use Guidelines for Canada (LRCUG): A narrative review of evidence and recommendations. Can J Public Health 2011; 102: 324-327

[55] Fischer B, Russell C, Brink Wvd et al. Lower-Risk Cannabis Use Guidelines (LRCUG): A comprehensive update of evidence and recommendations. American Journal of Public Health epub, doi: 10.2105/AJPH.2017.30381

[56] Swift W, Copeland J, Lenton S. Cannabis and harm reduction. Drug and alcohol review 2000; 19: 101-112

[57] Grotenhermen F. Harm reduction associated with inhalation and oral administration of cannabis and THC. Journal of Cannabis Therapeutics 2001; $1: 133-152$

[58] Hathaway AW, Erickson PG. Drug reform principles and policy debates: harm reduction prospects for cannabis in Canada. Journal of Drug Issues 2003; 33: 465-495

[59] Kerr WC, Stockwell T. Understanding standard drinks and drinking guidelines. Drug and alcohol review 2012; 31: 200-205

[60] Casajuana C, López-Pelayo H, Balcells MM et al. Working on a standard joint unit: A pilot test. Adicciones 2016, Doi: 10.20882/adicciones.721 2016; doi: 10.20882/adicciones.721, Publish Date: 2016 Sep 29

[61] Ridgeway G, Kilmer B. Bayesian inference for the distribution of grams of marijuana in a joint. Drug \& Alcohol Dependence 2016; 165 : $175-180$

[62] Wetherill RR, Hager N, Guthier E et al. Gram Years: A method to standardize and quantify lifetime cannabis consumption. Cannabis and cannabinoid research 2016; 1: 216-217

[63] Di Forti M, Marconi A, Carra E et al. Proportion of patients in south London with first-episode psychosis attributable to use of high potency cannabis: a case-control study. The Lancet Psychiatry 2015; 2: 233-238

[64] Coyne J. Cannabis and psychosis. The Lancet Psychiatry 2015; 2: 380-381

[65] Abrams DI, Vizoso HP, Shade SB et al. Vaporization as a smokeless cannabis delivery system: a pilot study. Clinical pharmacology and therapeutics 2007; 82: 572-578

[66] Gieringer DH. Cannabis "Vaporization": A Promising Strategy for Smoke Harm Reduction. Journal of Cannabis Therapeutics 2001; 1 : 153-170

[67] Earleywine M, Barnwell SS. Decreased respiratory symptoms in cannabis users who vaporize. Harm Reduct J 2007; 4: 11

[68] Gartner CE. Mull it over: cannabis vaporizers and harm reduction. Addiction 2015; 110: 1709-1710

[69] Malouff JM, Rooke SE, Copeland J. Experiences of marijuana-vaporizer users. Substance abuse 2014; 35: 127-128 
[70] Tashkin DP. How benefecial is vaping cannabis to respiratory health compared to smoking? Addiction 2015; 110: 1706-1707

[71] Budney AJ, Sargent JD, Lee DC. Vaping cannabis (marijuana): parallel concerns to e-cigs? Addiction 2015; 110: 1699-1704

[72] Fischer B. Cannabis vaping and public health - some comments on relevance and implications. Addiction 2015; 110: 1705-1706

[73] Dubois S, Mullen N, Weaver B et al. The combined effects of alcohol and cannabis on driving: Impact on crash risk. Forensic science international 2015; 248: 94-100

[74] Piontek D, Kraus L, Klempova D. Short scales to assess cannabis-related problems: a review of psychometric properties. Substance abuse treatment, prevention, and policy 2008; 3: 25

[75] Faggiano F, Minozzi S, Versino E et al. Universal school-based prevention for illicit drug use. The Cochrane database of systematic reviews 2014, doi:10.1002/14651858.CD003020.pub3: CD003020

[76] Teesson M, Newton NC, Barrett EL. Australian school-based prevention programs for alcohol and other drugs: a systematic review. Drug and alcohol review 2012; 31: 731-736

[77] Champion KE, Newton NC, Barrett EL et al. A systematic review of school-based alcohol and other drug prevention programs facilitated by computers or the internet. Drug and alcohol review 2013; 32: $115-123$

[78] Porath-Waller AJ, Beasley E, Beirness D]. A meta-analytic review of school-based prevention for cannabis use. Health education \& behavior: the official publication of the Society for Public Health Education 2010; 37: 709-723

[79] Norberg MM, Kezelman S, Lim-Howe N. Primary prevention of cannabis use: a systematic review of randomized controlled trials. PloS one 2013; 8: e53187

[80] Vogl LE, Newton NC, Champion KE et al. A universal harm-minimisation approach to preventing psychostimulant and cannabis use in adolescents: a cluster randomised controlled trial. Substance abuse treatment, prevention, and policy 2014; 9:

[81] Newton NC, Teesson M, Vogl LE et al. Internet-based prevention for alcohol and cannabis use: final results of the Climate Schools course. Addiction 2010; 105: 749-759

[82] Midford R, Cahill H, Foxcroft D et al. Drug education in Victorian schools (DEVS): the study protocol for a harm reduction focused school drug education trial. BMC public health 2012; 12: 112

[83] Hawthorne G, Garrard J, Dunt D. Does Life Education's drug education programme have a public health benefit? Addiction 1995; 90: 205-215

[84] Bonomo YA, Bowes G. Putting harm reduction into an adolescent context. Journal of Paediatrics and Child Health 2001; 37: 5-8

[85] Midford R. Does drug education work? Drug and alcohol review 2000; 19: 441-446

[86] Hawthorne G. Drug education: myth and reality. Drug and alcohol review 2001; 20: 111-119

[87] Tupper KW. Teaching teachers to just say "know": Reflections on drug education. Teaching and Teacher Education 2008; 24: 356-367

[88] Fischer B, Jones W, Shuper P et al. 12-month follow-up of an exploratory 'brief intervention' for high-frequency cannabis users among Canadian university students. Substance abuse treatment, prevention, and policy 2012; 7 (15)

[89] Babor TF, McRee BG, Kassebaum PA et al. Screening, Brief Intervention, and Referral to Treatment (SBIRT): toward a public health approach to the management of substance abuse. Substance abuse 2007; 28: 7-30

[90] O’Donnell A, Anderson P, Newbury-Birch D et al. The impact of brief alcohol interventions in primary healthcare: a systematic review of reviews. Alcohol Alcohol 2014; 49: 66-78

[91] Kaner EF, Beyer F, Dickinson HO et al. Effectiveness of brief alcohol interventions in primary care populations. The Cochrane database of systematic reviews 2007, doi:10.1002/14651858.CD004148.pub3: CD004148

[92] World Health Organization. Global strategy to reduce the harmful use of alcohol. Geneva, Switzerland: WHO Press; 2010

[93] Schaub MP, Wenger A, Berg $O$ et al. A web-based self-help intervention with and without chat counseling to reduce cannabis use in problematic cannabis users: three-arm randomized controlled trial. Journal of medical Internet research 2015; 17: e232

[94] Copeland J, Swift W, Roffman R et al. A randomized controlled trial of brief cognitive-behavioral interventions for cannabis use disorder. Journal of substance abuse treatment 2001; 21: 55-64

[95] Benard V, Rolland B, Messaadi N et al. Cannabis use: what to do in general practice? Presse medicale 2015; 44: 707-715

[96] Potier C, Laprevote V, Dubois-Arber F et al. Supervised injection services: what has been demonstrated? A systematic literature review. Drug and alcohol dependence 2014; 145: 48-68

[97] Martin-Santos R, Crippa JA, Batalla A et al. Acute Effects of a Single, Oral Dose of D9-Tetrahydrocannabinol (THC) and Cannabidiol (CBD) Administration in Healthy Volunteers. Curr Pharm Des 2012; 18 : 4966-4979

[98] Iseger TA, Bossong MG. A systematic review of the antipsychotic properties of cannabidiol in humans. Schizophrenia research 2015; 162: 153-161

[99] Crepault JF, Rehm J, Fischer B. The Cannabis Policy Framework by the Centre for Addiction and Mental Health: A proposal for a public health approach to cannabis policy in Canada. The International journal on drug policy 2016, doi:10.1016/j.drugpo.2016.04.013

[100] Task Force on Cannabis Legalization and Regulation. A Framework for the Legalization and Regulation of Cannabis in Canada. In: Canada H. ed.Ottawa: Health Canada; 2016

[101] Government of Canada. Legalizing and strictly regulating cannabis: the facts. Ottawa: 2017

[102] Schroers A. Drug-Checking - ein sichereres Verfahren für HarmReduction und Monitoring? In: Gaßman R, Bartsch G, Kepp J, (Hrsg.). Jahrburch Sucht 2015. Hamm: Deutsche Hauptstelle für Suchtfragen; 2015

[103] Brunt TM, Niesink RJM. The Drug Information and Monitoring System (DIMS) in the Netherlands: Implementation, results, and international comparison. Drug testing and analysis 2011; 3: 621-634

[104] European Monitoring Centre for Drugs and Drug Addiction. An inventory of on-site pill-testing interventions in the EU. In: Burkhart G. ed. Lisbon: EMCDDA; 2001

[105] Brunt TM, Nagy C, Bucheli A et al. Drug testing in Europe: monitoring results of the Trans European Drug Information (TEDI) project. Drug testing and analysis 2016, doi:10.1002/dta.1954

[106] Hungerbuehler I, Buecheli A, Schaub M. Drug Checking: A prevention measure for a heterogeneous group with high consumption frequency and polydrug use - evaluation of zurich's drug checking services. Harm Reduction Journal 2011; 8 (16)

[107] Pijlman FT, Rigter SM, Hoek J et al. Strong increase in total delta-THC in cannabis preparations sold in Dutch coffee shops. Addiction biology 2005; 10: 171-180

[108] Rigter S, Niesink R. THC-concentraties in wiet, nederwiet en hasj in Nederlandse coffeeshops (2015-2016)

[109] Shanahan M, Ritter A. Cost benefit analysis of two policy options for cannabis: status quo and legalisation. PloS one 2014; 9: e95569

[110] Apfel F. Cannabis - From Prohibition to Regulation: "When the music changes so does the dance"

[111] Stevenson B. Drug policy, criminal justice and mass imprisonment. Geneva: Global comission on drug policies; 2011

[112] Wodak A, Reinarman C, Cohen PDA. Cannabis control: costs outweigh the benefits. Br Med J 2002; 324: 105-108 
[113] Hall W, Lynskey M. Evaluating the public health impacts of legalizing recreational cannabis use in the USA. Addiction 2016, doi:10.1111/ add. 13428

[114] Hall W, Lynskey M. The challenges in developing a rational cannabis policy. Current opinion in psychiatry 2009; 22: 258-262

[115] Die Drogenbeauftragte der Bundesregierung. Nationale Strategie zur Drogen- und Suchtpolitik. Berlin: Bundesministerium für Gesundheit; 2012

[116] European Council. Drugs Strategy.In: General Secretariat of the Council ed, 2013-2020. Brussel: European Council; 2013

[117] Deutsche Hauptstelle für Suchtfragen. Cannabis Basisinformationen. Im Internet: http://www.dhs.de/fileadmin/user_upload/pdf/ Broschueren/Basisinfo_Cannabis.pdf; Zugriff: 28.11.2016

[118] Therapieladen e.V. Cannabis denn Sünde sein. Eine Broschüre rund ums Kiffen. Mit dem ersten Kiffertest. In. Berlin: 1998

[119] Fischer B. Drugs, Communities, and "Harm Reduction" in Germany: The new relevance of "Public Health". Principles in local responses Journal of Public Health Policy 1995; 16: 389-411

[120] Hanewinkel R, Wiborg G. Effektivität verhaltenspräventiver Interventionen zur Suchtvorbeugung. Suchttherapie 2003; 4: 183-191

[121] Kim J-I. Paradigmenwechsel in der Suchtprävention: akzeptierende Drogenerziehung. Theorie und Praxis der sozialen Arbeit 2004; 55: $18-25$

[122] Kraus L, Pabst A, Piontek D. Europäische Schülerstudie zu Alkohol und anderen Drogen 2011 (ESPAD). München: Institut für Therapieforschung; 2011

[123] Behrendt S, Wittchen HU, Hofler M et al. Transitions from first substance use to substance use disorders in adolescence: is early onset associated with a rapid escalation? Drug and alcohol dependence 2009; 99: 68-78

[124] Klein M. Geschichte der Suchtprävention. In: Hoff T, Klein M, (Hrsg.). Evidenzbasierung in der Suchtprävention: Möglichkeiten und Grenzen in Praxis und Forschung. Berlin: Springer; 2015: 37-42

[125] Barsch G. Drogenerziehung in der Praxis. In: Barsch G.ed Projektideen zur Förderung der Drogenmündigkeit. Weinheim: Beltz Juventa; 2016

[126] Wolstein J. Evidenzbasierte Medizin: Vorbild für die Suchtprävention? In: Hoff T, Klein M, (Hrsg.). Evidenzbasierung in der Suchtprävention Möglichkeiten und Grenzen in Praxis und Forschung. Berlin: Springer; 2015: 57-65

[127] Midford R. Drug prevention programmes for young people: where have we been and where should we be going? Addiction 2010; 105: 1688-1695

[128] Bonnet U, Harries-Hedder K, Leweke FM et al. AWMF-Leitlinie: Cannabisbezogene Störungen. Fortschr Neurol Psychiat 2004; 72: 318-329

[129] Hildebrand A, Sonntag D, Bauer C et al. Versorgung Suchtkranker in Deutschland: Ergebnisse der Suchthilfestatistik 2007. Sucht 2009; 55 : 15-34

[130] Brand H, Künzel J, Pfeiffer-Gerschel T et al. Cannabisbezogene Störungen in der Suchthilfe: Inanspruchnahme, Klientel und Behandlungserfolg. Sucht 2016; 62: 9-21
[131] Görgen W, Hartmann R, Oliva H. Frühintervention bei erstauffälligen Drogenkonsumenten - FreD: Abschlussbericht der wissenschaftlichen Begleitung.

[132] Tossmann P, Kasten L. Realize it - Abschlussbericht des Bundestransfers von "Realize it“.

[133] Tossmann HP, Jonas B, Tensil MD et al. A controlled trial of an internet-based intervention program for cannabis users. Cyberpsychology, behavior and social networking 2011; 14: 673-679

[134] Cousto H, Harrach T, Kollwitz S et al. Drug-Checking-Konzept für die Bundesrepublik Deutschland. In: Cousto H, Harrach T, Hentschel A et al. (eds). Konzeptioneller Vorschlag zur Organisation von Drug-Checking. Berlin: techno-netzwerk berlin; 2000

[135] Bundeskriminalamt. Polizeiliche Kriminalstatistik für das Jahr 2016. In: Bundeskriminalamt ed; 2017

[136] Cousto H, Stöver H. Repression und kein Ende?! Eine Würdigung der aktuellen polizeilichen Zahlen zur Kriminalisierung von Drogengebraucher_innen. In 4 Alternativer Drogen- und Suchtbericht 2017. Lengerich, Germany: Pabst Science Publishers; 2017

[137] Schäfer C, Paoli L. Drogen und Strafverfolgung: Die Anwendung des § 31 a BtMG und anderer Opportunitätsvorschriften auf Drogenkonsumentendelikte. Freiburg. 2006

[138] Böllinger L, Quensel S. Drugs and driving: Dangerous youths or anxious adults? Journal of Drug Issues 2002; 32: 553-566

[139] Wong K, Brady JE, Li G. Establishing legal limits for driving under the influence of marijuana. Injury Epidemiology 2014; 1(26)

[140] Grotenhermen F, Leson G, Berghaus G et al. Developing limits for driving under cannabis. Addiction 2007; 102: 1910-1917

[141] Nutt D], King LA, Phillips LD et al. Drug harms in the UK: a multicriteria decision analysis. Lancet 2010; 376: 1558-1565

[142] Singleton N, Strang J. What would an evidence based drug policy be like? Bmj 2014; 349: g7493

[143] Stafford N. Germany considers legalising cannabis for medical use. Bmj 2015; 351: h5952

[144] Rehm J, Shield KD, Rehm MX et al. Alcohol consumption, alcohol dependence, and attributable burden of disease in Europe: potential gains from effective interventions for alcohol dependence. Toronto, Canada: Centre for Addiction and Mental Health; 2012

[145] Piontek D, Kraus L, Bjarnason T et al. Individual and country-level effects of cannabis-related perceptions on cannabis use. A multilevel study among adolescents in 32 European countries. The Journal of adolescent health: official publication of the Society for Adolescent Medicine 2013; 52: 473-479

[146] Hindocha C, Freeman TP, Ferris JA et al. No Smoke without Tobacco: A global overview of cannabis and tobacco routes of administration and their association with intention to quit. Frontiers in psychiatry 2016; 7: 104

[147] Lau N, Sales P, Averill S et al. Responsible and controlled use: Older cannabis users and harm reduction. The International journal on drug policy 2015 ; 26 : 709-718 